Draft Version August 12, 2018

Preprint typeset using $\mathrm{LAT}_{\mathrm{E}} \mathrm{X}$ style AASTeX6 v. 1.0

\title{
EVIDENCE FOR A DUSTY DARK DWARF GALAXY IN THE QUADRUPLE LENS MG0414+0534
}

\author{
KaIKI TARo InOUe \\ Faculty of Science and Engineering, Kindai University, Higashi-Osaka, 577-8502, Japan \\ SATOKi Matsushita \\ Academia Sinica Institute of Astronomy and Astrophysics, P.O. Box 23-141, Taipei 10617, Taiwan, Republic of China \\ TAKeo Minezaki \\ Institute of Astronomy, School of Science, University of Tokyo, Mitaka, Tokyo 181-0015, Japan \\ Masashi Chiba \\ Astronomical Institute, Tohoku University, Aoba-ku, Sendai 980-8578, Japan
}

\begin{abstract}
We report the $4 \sigma$ detection of a faint object with a flux of $\sim 0.3 \mathrm{mJy}$, in the vicinity of the quadruply lensed QSO MG 0414+0534 using the Atacama Large Millimeter/submillimeter array (ALMA) Band 7. The object is most probably a dusty dark dwarf galaxy, which has not been detected in either the optical, near-infrared (NIR) or radio ( $\mathrm{cm}$ ) bands. An anomaly in the flux ratio of the lensed images observed in Band 7 and the mid-infrared (MIR) band and the reddening of the QSO light color can be simultaneously explained if we consider the object as a lensing substructure with an ellipticity $\sim 0.7$ at a redshift of $0.5 \lesssim z \lesssim 1$. Using the best-fit lens models with three lenses, we find that the dark matter plus baryon mass associated with the object is $\sim 10^{9} M_{\odot}$, the dust mass is $\sim 10^{7} M_{\odot}$ and the linear size is $\gtrsim 5 \mathrm{kpc}$. Thus our findings suggest that the object is a dusty dark dwarf galaxy. A substantial portion of faint submillimeter galaxies (SMGs) in the universe may be attributed to such dark objects.
\end{abstract}

Keywords: gravitational lensing: strong — galaxies: dwarf

\section{INTRODUCTION}

The flux ratios of lensed images in some quadruply lensed QSOs disagree with the prediction of best-fit lens models with a smooth potential whose fluctuation scale is larger than the separation between the lensed images. Such a discrepancy is called the "anomalous flux ratio" and has been considered as an imprint of cold dark matter subhalos with a mass of $\sim 10^{8-9} M_{\odot}$ in the lensing galaxy (Mao \& Schneider 1998; Chiba 2002; Dalal \& Kochanek 2002; Metcalf \& Zhao 2002; Kochanek \& Dalal 2004; Metcalf et al. 2004; Chiba et al. 2005; Sugai et al. 2007; More et al. 2009; Minezaki et al. 2009; Xu et al. 2009, 2010). Subhalos can be also detected through their effects on the image positions (Ros et al. 2000; Inoue \& Chiba 2005a,b; Koopmans 2005; Vegetti et al. 2012, 2014; Hezaveh et al. 2016).

However, the flux-ratio anomalies can be also explained by the weak lensing effects due to intervening halos and voids with a mass scale of $\lesssim 10^{9} M_{\odot}$ in the line-of-sight (Metcalf 2005; Xu et al. 2012; Inoue \& Takahashi 2012; Takahashi \& Inoue 2014; Inoue 2015; Inoue et al. 2016).
For lens systems with a source at a high redshift $z \gtrsim 2$, Inoue (2016) has recently argued that the main cause of the anomaly is structures in the intervening line-of-sights rather than subhalos associated with the lensing galaxy. In order to measure the redshift of possible perturbers, we need to observe the flux ratios and positions of lensed images as precisely as possible.

In this paper, we present our result on Atacama Large Millimeter/submillimeter Array (ALMA) continuum observations of the quadruply lensed, radio-loud QSO MG 0414+0534. It has been known that MG 0414+0534 shows a strong sign of anomaly in the flux ratio and reddening in the optical and near-infrared (NIR) band (see Minezaki et al. 2009 and references therein). The origin of these features has not been fully understood yet. Throughout this paper, we use the Planck 2016 cosmological parameters: $h=0.678, \Omega_{m}=0.308$ and $\Omega_{\Lambda}=0.692$ (Planck Collaboration et al. 2016).

\section{OBSERVATIONS AND DATA REDUCTION}

The observations of MG $0414+0534$ were carried out with ALMA at Band 7 on 2015 June 13 and August 14 as 
part of Cycle 2 (Project ID: 2013.1.01110.S, PI: K.T. Inoue). The number of antennas used in the observation in June was 35 and that in August was 42. The phase center was $\alpha=04^{\mathrm{h}} 14^{\mathrm{m}} 37^{\mathrm{s}} .7686, \delta=+05^{\circ} 34^{\prime} 42^{\prime \prime} \cdot 352$ (J2000). The total on-source integrating times were $104.63 \mathrm{~min}$ utes and 52.28 minutes and the recoverable largest angular size was $\sim 3.3 \mathrm{arcsec}$. The ALMA correlator was configured to have four spectral windows centered at $335.0 \mathrm{GHz}, 337.0 \mathrm{GHz}, 347.0 \mathrm{GHz}$ and $349.0 \mathrm{GHz}$ with a bandwidth $2 \mathrm{GHz}$ and a frequency width of $15.625 \mathrm{MHz}$ for each window.

The calibration and imaging of the data were carried out using the Common Astronomy Software Application package (CASA; McMullin et al. 2007). The continuum images produced with a natural or a robust (robust $=-1$ ) weighting of the visibilities were used for imaging and modeling, respectively. We used multi-scale imaging (Cornwell 2008) with scales of 0, 6, 12, 24 and 48 for the natural weighting (pixel size is $0.02 \mathrm{arcsec}$ ) and $0,6,12$ and 24 for the robust (robust $=-1$ ) weighting (pixel size is 0.01 arcsec). The images have not been corrected for the primary beam attenuation because the source is small and located at the center of the primary beam. The $1 \sigma$ noises in the flux density per pixel are $49 \mu \mathrm{Jy}$ per beam and $140 \mu \mathrm{Jy}$ per beam, the full widths at half maximum of the elliptical gaussian fitted to the dirty beam are $\left(0^{\prime \prime} .278 \times 00^{\prime \prime} .243\right)$ and $\left(0^{\prime \prime} 135 \times 00^{\prime \prime} 111\right)$ and the PA's measured in degrees East of North are 46.5 and $75^{\circ}$, for the natural and robust (robust $=-1$ ) weightings, respectively.

\section{RESULTS}

\subsection{Dust continuum emission}

The dust continuum (Barvainis \& Ivison 2002) image obtained with a natural weighting (Figure 1, left) shows bright quadruply lensed spots A1, A2, B and C and a faint Einstein ring. As shown, lensed spots A1, $\mathrm{A} 2$ and $\mathrm{B}$ are resolved as separate regions if a robust $r=-1$ weighting is used. The aperture flux ratios ${ }^{1}$ for a robust $r=-1$ weighting were $(\mathrm{A} 2 / \mathrm{A} 1, \mathrm{~B} / \mathrm{A} 1)=$ $(0.87 \pm 0.03,0.38 \pm 0.06)$. Note that the errors were estimated using the flux density in apertures of the same size placed at source-free locations. We assumed that correlations between errors are negligible. The obtained result is consistent with the flux ratios in the MIR band $(\mathrm{A} 2 / \mathrm{A} 1, \mathrm{~B} / \mathrm{A} 1, \mathrm{C} / \mathrm{A} 1)=(0.919 \pm$ $0.021,0.347 \pm 0.013,0.139 \pm 0.014$ ) (Minezaki et al. 2009; MacLeod et al. 2013).

Interestingly, the image obtained with a natural weighting showed a faint object in the vicinity of A2, which we call 'object $\mathrm{Y}$ ' (shown in a red circle in Fig-

\footnotetext{
${ }^{1}$ Within an aperture radius of 0.21 arcsec, the neighboring pixels with flux density $>3.5 \sigma$ are all included.
}

ure 1, left). The apparent angular size is equal to or smaller than the beam size. The flux density of $\mathrm{Y}$ inside an aperture radius of 0.26 arcsec centered at the peak is $0.26 \mathrm{mJy}$ and the locally maximum value for the aperture flux is $0.27 \mathrm{mJy}$. The corresponding statistical significances, which were estimated using the flux density in apertures with the same size placed at source-free locations, were $4.0 \sigma$ and $4.1 \sigma$, respectively. A gaussian fit to the intensity of the faint emission yielded a peak flux density of $0.2 \mathrm{mJy} /$ beam corresponding to $4.0 \sigma$.

Assuming a dust mass opacity coefficient, $\kappa_{850 \mu \mathrm{m} /\left(1+z_{\mathrm{Y}}\right)}=0.077\left(1+z_{\mathrm{Y}}\right)^{\beta} \mathrm{m}^{2} \mathrm{~kg}^{-1}$ (Dunne et al. 2000), for an emissivity index $\beta=1-2$ with a dust temperature $T_{\mathrm{d}}=20-50 \mathrm{~K}$ and a dust redshift $z_{\mathrm{Y}}=0.5-1$ (see subsection 3.2), the dust mass is $M_{\mathrm{d}}=10^{6-7} M_{\odot}$. In the following, we interpret $\mathrm{Y}$ as the central core of a dusty dwarf galaxy.

\subsection{Flux-Ratio Anomaly}

The source of MG0414+0534, at a redshift of $z_{\mathrm{S}}=$ 2.639 , is lensed by a foreground elliptical galaxy G (the primary lens) at $z_{\mathrm{L}}=0.9584$ (Hewitt et al. 1992; Lawrence et al. 1995; Tonry \& Kochanek 1999). It has been known that a canonical model, a singular isothermal ellipsoid plus an external shear (SIE-ES) does not give a good fit. In order to improve the fit, we need to take into account a possible satellite galaxy, object X, which was detected in the I band HST/NICMOS image (Schechter \& Moore 1993; Ros et al. 2000; Trotter et al. 2000). However, the observed mid-infrared (MIR) flux ratio of A1 to A2 is still not consistent with the SIE-ES$\mathrm{X}$ model prediction even if low-multipole modes $m=3,4$ are added to the potential. If a subhalo resides in the vicinity of $\mathrm{A} 2$, the anomalous flux ratio $\mathrm{A} 1 / \mathrm{A} 2$ can be explained (Minezaki et al. 2009; MacLeod et al. 2013).

First, we used a canonical model, an SIE-ES for G plus a cored singular isothermal sphere (cored-SIS) for $\mathrm{X}$. The model has 13 parameters: the position $\left(x_{\mathrm{G}}, y_{\mathrm{G}}\right)$, effective Einstein angular radius $b_{\mathrm{G}}{ }^{2}$, ellipticity $e_{\mathrm{G}}$ and position angle $\phi_{\mathrm{G}}$ for $\mathrm{G}$, the position $\left(x_{\mathrm{X}}, y_{\mathrm{X}}\right)$, effective Einstein angular radius $b_{\mathrm{X}}$, core radius $r_{\mathrm{X}}$ for $\mathrm{X}$, the amplitude $\gamma$ and position angle $\phi_{\gamma}$ (direction of the associated mass clump) of the external shear and the source position $\left(x_{s}, y_{s}\right)\left({ }^{\prime \prime}\right)$. All the position angles are measured in degrees East of North. The redshift $z_{\mathrm{X}}$ of $\mathrm{X}$ was assumed to be $z_{\mathrm{X}}=z_{\mathrm{L}}$ (MacLeod et al. 2013) ${ }^{3}$. We used the measured HST WFPC/WFPC2 positions of lensed images and centroids of lensing galaxies, $\mathrm{G}$ and $\mathrm{X}$ in the CASTLES data archive $^{4}$ (Falco et al. 1997), and the MIR flux ratios, A2/A1 and B/A1 (Minezaki et al. 2009;

\footnotetext{
${ }^{2}$ We took a definition of $b_{G}$ for which the mass inside $b_{G}$ coincides with that inside the critical curve (Kormann et al. 1994).

${ }^{3}$ We found that a deviation of $z_{\mathrm{X}}$ from $z_{\mathrm{L}}$ yields a worse fit.

${ }^{4}$ http://www.cfa.harvard.edu/castles/
} 

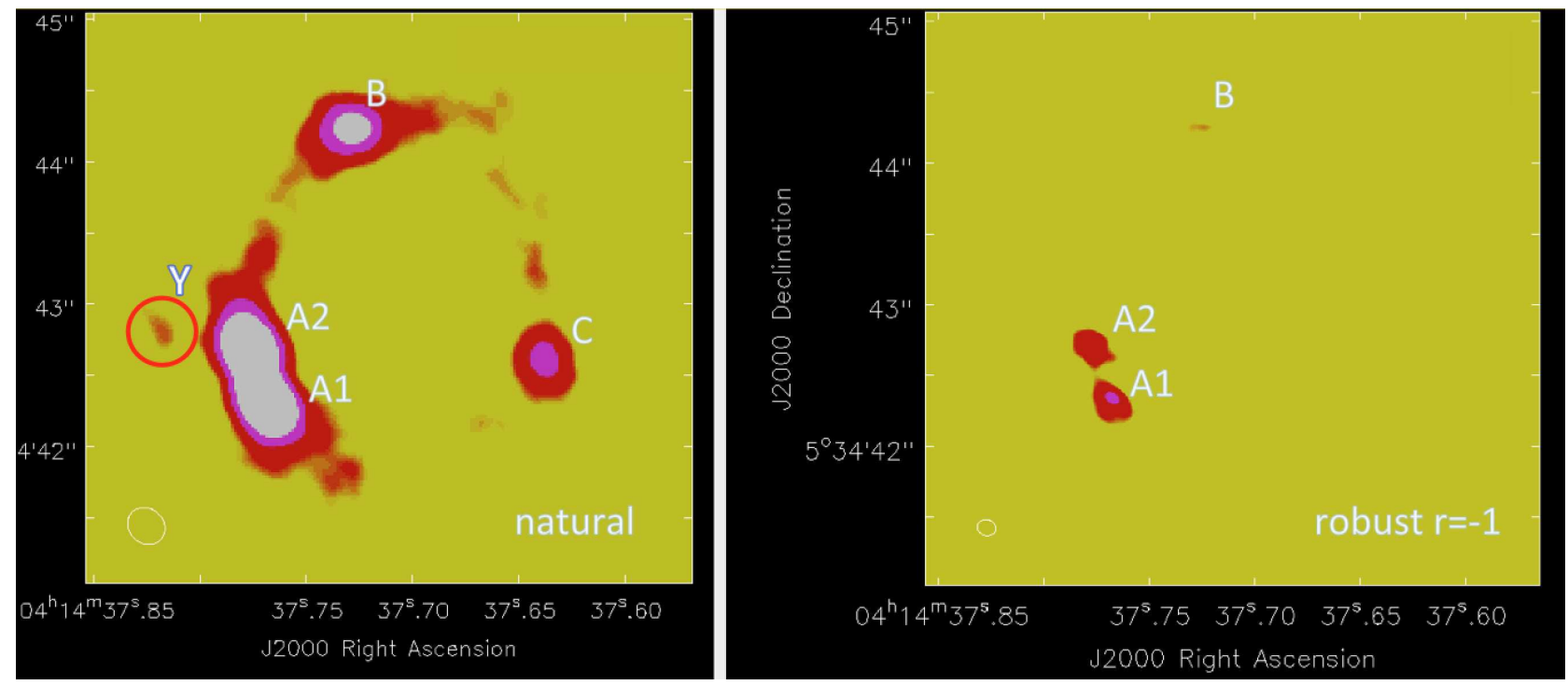

Figure 1. ALMA $0.88 \mathrm{~mm}$ (Band 7) dust continuum images of MG0414+0534. The surface brightness $(\gtrsim 4 \sigma)$ is plotted in color for a natural weighting (left) and a robust (robust $=-1$ ) weighting (right). The sizes of a Gaussian fitted beam, which are shown in the bottom-left corner of each image are $278 \times 243$ mas and $135 \times 111$ mas, respectively. A faint spot inside a red circle (left) is an object $Y$.
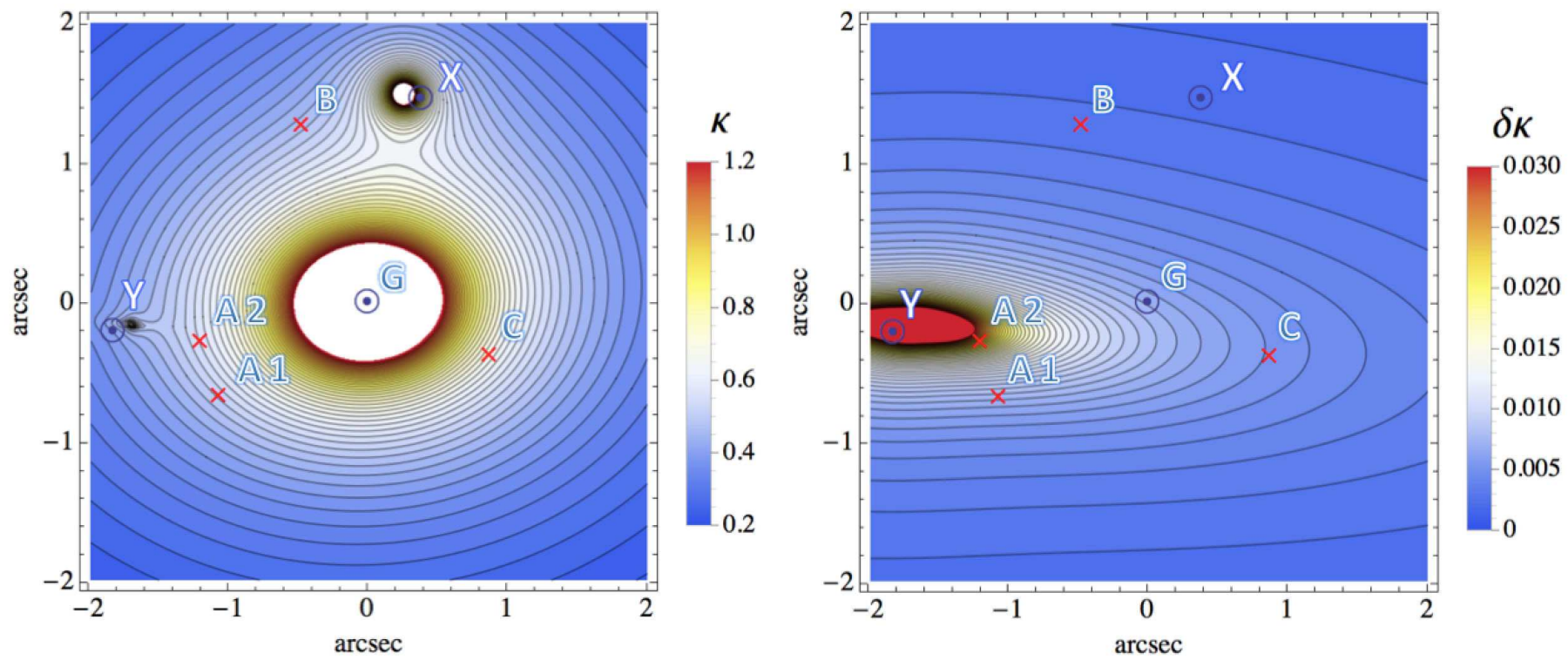

Figure 2. The total convergence $\kappa$ (left) and the convergence contribution $\delta \kappa$ from object Y (right) for a best-fit lens model with $e_{\mathrm{Y}}=0.72$ and $z_{\mathrm{Y}}=0.548$. Circled dots represent the positions of the observed centroids of the primary lensing galaxy G, object X and object Y. Red crosses represent the position of the lensed HST images of MG 0414+0534 (Falco et al. 1997). The surface mass density at A2 is approximately three times larger than that at A1.

MacLeod et al. 2013). Note that MIR and submillimeter fluxes are free from microlensing by stars and dust extinction whereas optical and NIR fluxes are potentially influenced by these systematics.

We assumed a weak prior for the amplitude of the external shear $\gamma(=0.05 \pm 0.05)$ and the ellipticity $e_{\mathrm{G}}(=$ $0 \pm 1$ ). We also assumed an error of 0.2 arcsec for the position of the centroid of $\mathrm{X}$, which is roughly the angular size, and 0.003 arcsec for the position of the centroid of G. A large error for $\mathrm{X}$ was adopted because of possible contamination from the lensed fifth image. We had $\chi_{\text {tot }}^{2} /$ dof $=29.0 / 4$ with a best-fit flux ratio $\mathrm{A} 2 / \mathrm{A} 1=1.005$, which implies that the fit is not satisfactory (see Table 1 for the definition of $\chi_{\mathrm{tot}}^{2}$ ). 
Table 1. Modeling Results for MG 0414+0534

\begin{tabular}{lccc}
\hline \hline Model & $\mathrm{G}+\mathrm{X}$ & $\mathrm{G}+\mathrm{X}+\mathrm{Y}($ subhalo) & $\mathrm{G}+\mathrm{X}+\mathrm{Y}$ (line-of-sight) \\
\hline$b_{\mathrm{G}}\left({ }^{\prime \prime}\right)$ & 1.104 & 1.113 & 1.114 \\
$\left(x_{s}, y_{s}\right)\left({ }^{\prime \prime}\right)$ & $(-0.0663,0.2603)$ & $(-0.0919,0.2130)$ & $(-0.0950,0.2157)$ \\
$e_{\mathrm{G}}$ & 0.303 & 0.223 & 0.225 \\
$\phi_{\mathrm{G}}(\mathrm{deg})$ & -87.9 & -85.4 & -85.4 \\
$\gamma$ & 0.0878 & 0.0848 & 0.0862 \\
$\phi_{\gamma}(\mathrm{deg})$ & 47.5 & 54.7 & 54.7 \\
$\left(x_{\mathrm{G}}, y_{\mathrm{G}}\right)\left({ }^{\prime \prime}\right)$ & $(0.0020,-0.0011)$ & $(0.0006,-0.0001)$ & $(-0.0003,0.0006)$ \\
$b_{\mathrm{X}}\left({ }^{\prime \prime}\right)$ & 0.199 & 0.154 & 0.154 \\
$\left(x_{\mathrm{X}}, y_{\mathrm{X}}\right)\left({ }^{\prime \prime}\right)$ & $(0.390,1.513)$ & $(0.274,1.457)$ & $(0.256,1.495)$ \\
$r_{\mathrm{X}}\left({ }^{\prime \prime}\right)$ & 0.007 & 0.032 & 0.033 \\
$b_{\mathrm{Y}}\left({ }^{\prime \prime}\right)$ & & 0.008 & 0.015 \\
$\left(x_{\mathrm{Y}}, y_{\mathrm{Y}}\right)\left({ }^{\prime \prime}\right)$ & & $(-1.751,-0.1151)$ & $(-1.692,-0.152)$ \\
$e_{\mathrm{Y}}$ & & 0.72 & 0.72 \\
$\phi_{\mathrm{Y}}(\mathrm{deg})$ & & 85.74 & 85.73 \\
$z_{\mathrm{Y}}$ & & 0.958 & 0.548 \\
$\chi_{\text {pos }}^{2}$ & 6.35 & 1.42 & 2.50 \\
$\chi_{\text {flux }}^{2}$ & 22.09 & 3.41 & 2.93 \\
$\chi_{\text {weak }}^{2}$ & 0.57 & 0.57 & 0.57 \\
$\chi_{\text {tot }}^{2} / \mathrm{dof}$ & $29.0 / 4$ & $5.4 / 2$ & $6.0 / 2$ \\
$\mathrm{~A} 2 / \mathrm{A} 1$ & 1.005 & 0.925 & 0.932 \\
$\mathrm{~B} / \mathrm{A} 1$ & 0.342 & 0.350 & 0.351 \\
$\mathrm{C} / \mathrm{A} 1$ & 0.171 & 0.164 & 0.161 \\
\hline
\end{tabular}

Note $-\chi_{\text {tot }}^{2}$ is the sum of contributions from the image and lens positions $\chi_{\text {pos }}^{2}$, the flux ratios $\chi_{\text {flux }}^{2}$, and the weak priors $\chi_{\text {weak }}^{2}$ on $e_{\mathrm{G}}, \gamma$ and $e_{\mathrm{Y}}$ if applicable. The coordinates are centered at the centroid of the primary lensing galaxy G (CASTLES database) (Falco et al. 1997).

Next, we added an SIE for modeling Y. The parameters are the position $\left(x_{\mathrm{Y}}, y_{\mathrm{Y}}\right)$, effective Einstein angular radius $b_{\mathrm{Y}}$, ellipticity $e_{\mathrm{Y}}$, and position angle $\phi_{\mathrm{Y}}$. As a first guess, we assumed $z_{\mathrm{Y}}=z_{\mathrm{L}}$. The astrometric error of $\mathrm{Y}$ is assumed to be $0.13 \mathrm{arcsec}$, which is roughly the angular size. We obtained the coordinates of the centroid of $\mathrm{Y}\left(-1{ }^{\prime \prime} 824,-0{ }^{\prime \prime} 212\right)$ by matching the brightest spots in the ALMA and CASTLES data. The residual errors were $\sim 0.01$ arcsec.

Then, it turned out that the fit was greatly improved provided that the ellipticity $e_{\mathrm{Y}}$ of $\mathrm{Y}$ satisfies $e_{\mathrm{Y}} \gtrsim 0.7$. We obtained a better fit for a larger ellipticity $e_{Y}$. For explaining the reddening of A1 and A2 by dust extinction, however, very large ellipticity is not likely. Therefore, we assumed another weak prior on the ellipticity of $\mathrm{Y}$ as $e_{\mathrm{Y}}=0 \pm 1$, which results in 20 total constraints. For $e_{\mathrm{Y}}=0.72$, we had $\chi_{\text {tot }}^{2} /$ dof $=5.4 / 2$ with a best-fit flux ratio $\mathrm{A} 2 / \mathrm{A} 1=0.925$ (Table 1 ). For models with a larger ellipticity, the ratio of convergence of $\mathrm{Y}$ at $\mathrm{A} 1$ and $\mathrm{A} 2$ becomes smaller than $\sim 1 / 3$, and hence the convergence at A1 becomes too small to account for the reddening in optical/IR bands (see subsection 3.3).

We also examined the dependence of the fit on the redshift $z_{\mathrm{Y}}$ of $\mathrm{Y}$. We found that $\chi_{\mathrm{tot}}^{2}$ is nearly constant if $0.5 \lesssim z_{\mathrm{Y}} \lesssim z_{\mathrm{L}}$. For $e_{\mathrm{Y}}=0.72$, a condition that $\chi_{\text {tot }}^{2} /$ dof $\leq 6.0 / 2=3.0$ yielded $0.548 \leq z_{\mathrm{Y}} \leq 0.983$. In the best-fit model with $e_{\mathrm{Y}}=0.72$ and $z_{\mathrm{Y}}=0.958(0.548)$ (Table 1), the masses of $\mathrm{Y}$ are $1.3(1.1) \times 10^{9} M_{\odot}$ and $3.7(3.6) \times 10^{9} M_{\odot}$ for regions in which the convergence contribution from $\mathrm{Y}$ satisfies $\delta \kappa>\delta \kappa(\mathrm{A} 2)$ and $\delta \kappa>$ $\delta \kappa(\mathrm{A} 1)^{5}$, respectively and the one-dimensional velocity dispersion corresponding to $b_{\mathrm{Y}}$ is $\sigma_{\mathrm{Y}} \sim 25(28) \mathrm{km} \mathrm{s}^{-1}$.

As shown in Figure 2, the convergence contribution $\delta \kappa$ from $\mathrm{Y}$ is equal to or less than $\sim 5$ percent of the total convergence $\kappa \sim 0.5$ at $\mathrm{A} 1$ and $\mathrm{A} 2 . \delta \kappa(A 2)$ is approximately three times larger than $\delta \kappa(A 1)$. The projected distance between the best-fit centroid of $\mathrm{Y}$ and $\mathrm{A} 2$ is $7.1 \mathrm{kpc}$ for $z_{\mathrm{Y}}=0.958$ and $5.3 \mathrm{kpc}$ for $z_{\mathrm{Y}}=0.548$.

In order to test the possibility that object $\mathrm{Y}$ is a sidelobe response to A1 or A2, we simulated an ALMA observation using simalma command in CASA 4.5.3. We used the same antenna configurations and observation time as those in the real observations. The value of the precipitable water vapor was adjusted to match the observed noise in the final image. The source model was obtained from the ALMA continuum image for a robust $(r=-1)$ weighting using a linear combination of multiple lensed images with a magnification weighting (see

5 Each mass corresponds to a total mass within an elliptical aperture centered at the centroid of Y. 

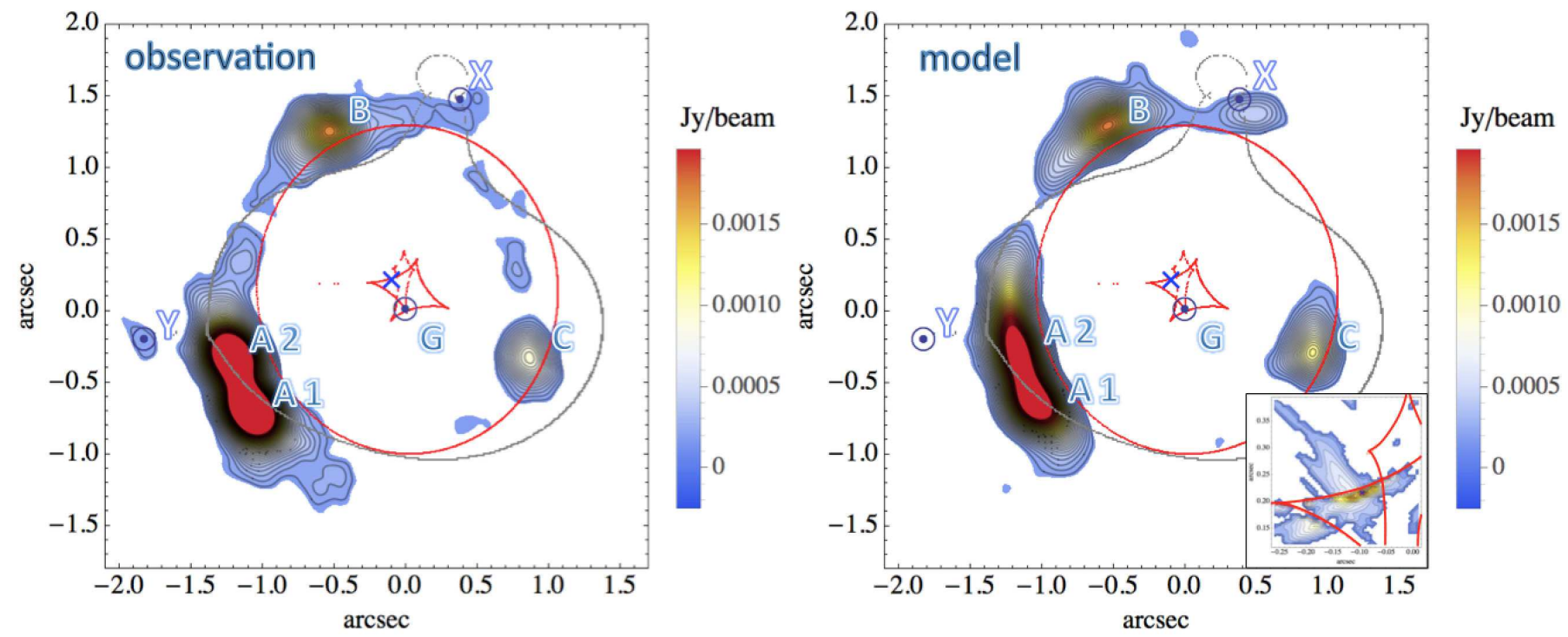

Figure 3. Observed and modeled dust continuum images for a three-galaxy model $(\mathrm{G}+\mathrm{X}+\mathrm{Y})$ with $e_{\mathrm{Y}}=0.72$ and $z_{\mathrm{Y}}=0.548$ (see Table 1). The both images were produced with a natural weighting. The color shows the surface brightness $(>3.5 \sigma)$ and the contour spacing is $1 \sigma$. The inset in the right panel shows the source model. The red curves are the caustics and cuts and the gray curves are the critical curves. The circled dots are the positions of the observed centroids of G, X and Y and the blue crosses represent the position of the QSO core in the source plane.

Inoue et al. 2016 for its definition). The total flux density was then adjusted to yield the observed value for a natural and a robust $(r=-1)$ weightings. We used the same multi-scale imaging as have been used for the corresponding real images. Here we assumed $e_{\mathrm{Y}}=0.72$ and $z_{\mathrm{Y}}=0.548$.

As shown in Figure 3, the feature of the quadruply lensed spots and arcs was successfully reproduced though minor difference remains. However, we were not able to find any spots around the position of $\mathrm{Y}$. Therefore, it is unlikely that $\mathrm{Y}$ is related with sidelobes of $\mathrm{A} 1$ or $\mathrm{A} 2$. The flux ratios within an aperture radius of $0.21 \mathrm{arcsec}$ were turned out to be $(\mathrm{A} 2 / \mathrm{A} 1, \mathrm{~B} / \mathrm{A} 1)=(0.85,0.52)$ for a robust $(r=-1)$ weighting, which are consistent with the observation of the dust continuum.

\subsection{Differential Extinction}

From the unusually red colors, it has been speculated that MG0414+0534 is obscured by dust (e.g., Hewitt et al. 1992). The optical and NIR flux ratios change with observing wavelength systematically, which can be explained by the different amounts of extinction by intervening dust in the line of sights. However, the primary lensing galaxy $\mathrm{G}$ is a passively evolving early-type galaxy, which is likely to be dust deficient (Tonry \& Kochanek 1999). Here we examine whether object Y can be the origin of the differential extinction.

Figure 4 shows the optical and near-infrared flux ratios of $\mathrm{A} 1 / \mathrm{B}, \mathrm{A} 2 / \mathrm{B}$, and $\mathrm{C} / \mathrm{B}$ in different wavelengths. The flux data were taken from the CASTLES data archive (Falco et al. 1997). The F555W-band data were not used because of their large photometric errors. Clear reddening trends are found in the flux ratios of $\mathrm{A} 1 / \mathrm{B}$ and $\mathrm{A} 2 / \mathrm{B}$ but not in $\mathrm{C} / \mathrm{B}$, which suggest that $\mathrm{A} 1$ and $\mathrm{A} 2$ are more obscured by intervening dust in the line of sight than $\mathrm{B}$ and $\mathrm{C}$, as has been reported in previous studies.

Then we fit extinction curves to the flux ratios of $\mathrm{A} 1 / \mathrm{B}$ and $\mathrm{A} 2 / \mathrm{B}$ in different wavelengths to estimate the amount of differential extinction. We adopted the dust extinction curve for the Small Magellanic Cloud (SMC) bar (Gordon et al. 2003) and assumed the MIR flux ratios as the intrinsic ones. In order to examine the dust extinction from a dwarf galaxy either in the intervening line of sight or in the galaxy G, we put a hypothetical dust obscurer at 0.5 , and 1.0 to estimate its amount of extinction, respectively. In most cases, the reduced $\chi^{2}$ for the fits of extinction curves were larger than unity if only the observational errors in the flux ratios are taken into account. To achieve unity for the reduced $\chi^{2}$, we added the root-sum-square of systematic errors. The amount of systematic errors was 0.01-0.04 dex.

In Figure 4, the best-fit extinction curves for A1/B and A2/B are overlaid on the flux-ratio data. The estimated differential extinctions of $\mathrm{A} 1$ relative to $\mathrm{B}$ were $0.33 \pm$ $0.03,0.23 \pm 0.01$ and those of $\mathrm{A} 2$ relative to $\mathrm{B}$ were $1.11 \pm$ $0.05,0.77 \pm 0.04$ for $0.5,1.0$, respectively. While the amount of extinction of the intervening obscurer becomes larger when its redshift become smaller, the flux ratios 


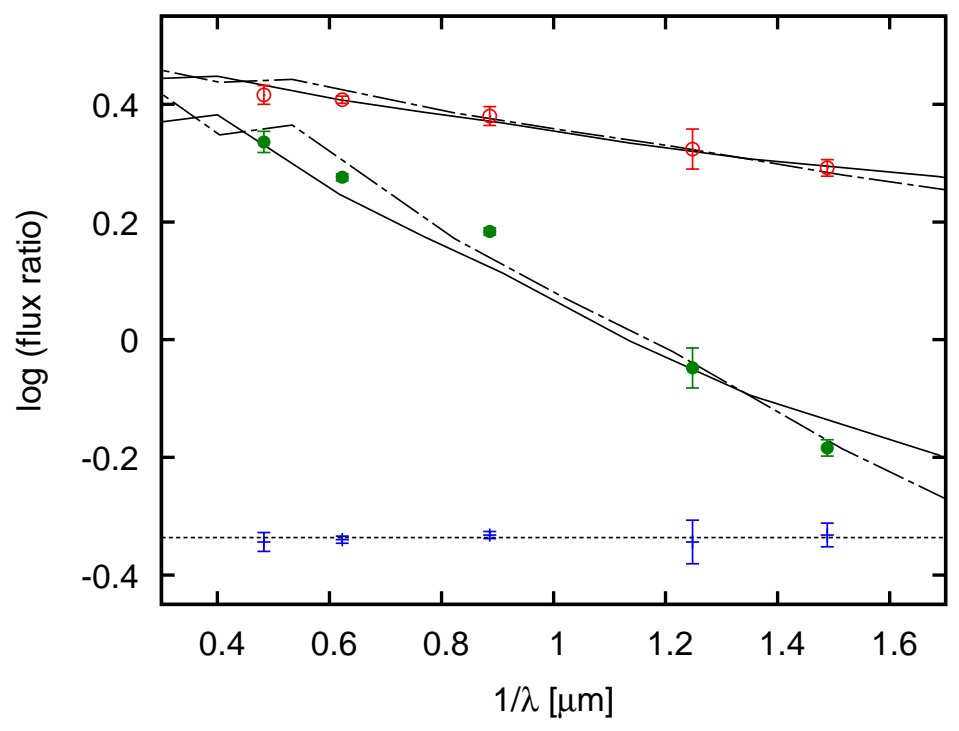

Figure 4. Optical and NIR flux ratios of MG 0414+0534 in different wavelengths. The open circles, the filled circles, and the plus marks represent the flux ratios of A1/B, A2/B, and C/B obtained from the CASTLES data archive, respectively (Falco et al. 1997). The dot-dashed, and the solid lines represent the best-fit extinction curves for the flux ratios of $\mathrm{A} 1 / \mathrm{B}$ and $\mathrm{A} 2 / \mathrm{B}$ in which the source of extinction is assumed to reside at $z=0.5$, and $z=1.0$, respectively. The dotted line represents the weighted average of the flux ratio of $\mathrm{C} / \mathrm{B}$.

are fitted reasonably well at any redshift assumed here. We note that our estimates of the differential extinction from an intervening obscurer at $z=1.0$ approximately agree with those in a previous study (Falco et al. 1999) in which the standard Galactic extinction law of $R_{\mathrm{V}}=3.1$ for $Y$ at $z_{\mathrm{d}}=z_{\mathrm{L}}=0.958$ is assumed.

The weighted average of the flux ratios of $\mathrm{C} / \mathrm{B}$ is also overlaid in Figure 4. The estimated value $0.461 \pm 0.002$ is consistent with the $\mathrm{C} / \mathrm{B}$ flux ratio in mid-infrared $(0.401 \pm 0.043)$ at 1.4 sigmas, and agrees well with those in our $\mathrm{G}+\mathrm{X}+\mathrm{Y}$ lens models (0.469 and 0.459) shown in Table 1.

We can estimate the ratio of the difference of convergence contribution $\delta \kappa$ between $\mathrm{A} 2$ and $\mathrm{B}$ to $\mathrm{A} 1$ and $\mathrm{B}$ as $(\delta \kappa(\mathrm{A} 2)-\delta \kappa(\mathrm{B})) /(\delta \kappa(\mathrm{A} 1)-\delta \kappa(\mathrm{B}))=4.3$ and 3.7 for the redshifts of $z_{\mathrm{Y}}=0.548$ and 0.958 , respectively (Figure 2). They are close to or slightly larger than the ratios of the differential extinction of $\mathrm{A} 2$ to that of $\mathrm{A} 1, \Delta A_{V}(\mathrm{~A} 2) / \Delta A_{V}(\mathrm{~A} 1) \approx 3.4$ for both the redshifts. Therefore, the large difference in the differential extinction of A2 relative to A1 can be naturally explained by our model in which object $\mathrm{Y}$ has an elongated SIE profile even though A1 is located close to A2.

The dust mass column density $\Sigma_{\text {dust }}$ in a sight line per an amount of extinction is $\Sigma_{\text {dust }} / A_{V}=$ $1.54 \times 10^{-1} M_{\odot} \mathrm{pc}^{-2} / A_{V}$ for the Galaxy model and $2.66 \times 10^{-1} M_{\odot} \mathrm{pc}^{-2} / A_{V}$ for the SMC dust model (Weingartner \& Draine 2001; Draine \& Li 2007). Assuming that $\mathrm{Y}$ resides at $z_{\mathrm{Y}}=z_{\mathrm{L}}$, the dust mass column density estimated from the amount of extinction in the site line of $\mathrm{A} 1(\mathrm{~A} 2)$ is $3.5(12) \times 10^{-2} M_{\odot} \mathrm{pc}^{-2}$ for the
Galaxy dust model, and $6.1(20) \times 10^{-2} M_{\odot} \mathrm{pc}^{-2}$ for the SMC dust model. If the dust mass distribution traces the best-fit SIE mass profile of $\mathrm{Y}$, then the estimated dust mass for the smallest elliptical region centered at the centroid of $\mathrm{Y}$ that includes $\mathrm{A} 1(\mathrm{~A} 2)$ is $1.6(0.6) \times 10^{7} M_{\odot}$ for the Galaxy dust model, and 2.7(1.1) $\times 10^{7} M_{\odot}$ for the SMC dust model. We find that the dust mass $\sim 10^{7} M_{\odot}$ estimated from the differential extinction does not significantly change if $z_{\mathrm{Y}}=0.5-1$ and it agrees with the value obtained from the sub-mm continuum emission of $\mathrm{Y}$ provided that the dust temperature is $T_{\mathrm{d}} \sim 20 \mathrm{~K}$, the emissivity index is $\beta=1-2$ and the redshift is $z_{\mathrm{Y}}=0.5-1$.

Thus these results indicate that object $\mathrm{Y}$ is a good candidate for an intervening object that causes the differential extinction of $\mathrm{A} 1$ and $\mathrm{A} 2$.

\section{DISCUSSIONS}

The detected 'object Y' is most probably a dusty dark dwarf galaxy at a redshift of $0.5 \lesssim z_{\mathrm{Y}} \lesssim 1$. If $\mathrm{Y}$ is associated with a subhalo at $z_{\mathrm{Y}} \sim z_{\mathrm{L}}$, then it may be also associated with $\mathrm{H}_{\mathrm{I}} 21-\mathrm{cm}$ absorption systems in the lensing galaxy $\mathrm{G}$, which has a large line-of-sight velocity $\sim 200 \mathrm{~km} \mathrm{~s}^{-1}$ (Allison et al. 2016). The inferred $\mathrm{H}_{\mathrm{I}}$ column density of the sum of three systems around $z=z_{\mathrm{L}}$ is $N_{\mathrm{H}_{\mathrm{I}}}=1.6 \times 10^{18}\left(T_{\mathrm{spin}} / f_{\mathrm{H}_{\mathrm{I}}}\right) \mathrm{cm}^{-2}$ (Curran et al. 2007), where $T_{\text {spin }}$ is the spin temperature and $f_{\mathrm{H}_{\mathrm{I}}}$ is the covering factor for the background continuum source. If we assume $T_{\text {spin }}=10^{3} \mathrm{~K}$ and $f_{\mathrm{H}_{\mathrm{I}}}=0.5$, then the $\mathrm{H}_{\mathrm{I}}$ column density estimated from absorption agrees with the value estimated from the difference in the hydrogen $\left(\mathrm{H}_{\mathrm{I}}+\mathrm{H}_{2}\right)$ column densities in sight lines to $\mathrm{A} 1$ and $\mathrm{A} 2$ 
relative to $\mathrm{B}, \Delta N_{\mathrm{H}_{\mathrm{I}}}=(3.3 \pm 1.0) \times 10^{21} \mathrm{~cm}^{-2}$, which was measured with the X-ray spectra (Dai et al. 2006). The latter can be converted into the sum of hydrogen mass column densities $\Sigma_{\mathrm{H}}$ in the sight lines to A1 and A2 as $\sim 2.6 \times 10^{1} M_{\odot} \mathrm{pc}^{-2}$. Then the sum of baryon mass column densities at the sight lines is estimated as $\sim 3.7 \times 10^{1} M_{\odot} \mathrm{pc}^{-2}$ assuming the Solar abundance (Asplund et al. 2009). This value is comparable to the corresponding surface mass density $\sim 3.2 \times 10^{1} M_{\odot} \mathrm{pc}^{-2}$ inferred from the best-fit SIE profile for Y. The hydrogen mass enclosing $\mathrm{A} 1(\mathrm{~A} 2)$ is then estimated as $\sim$ $3.1(1.1) \times 10^{9} M_{\odot}$ provided that the gas distribution traces the best-fit SIE profile. This implies that the mass profile of the lens model is solely determined by baryon which dominates over the dark matter. Such dark matter deficiency reminds us of 'missing dark matter' in some dwarf galaxies (Oman et al. 2016).

The dust to gas ratio in the sight line to $\mathrm{A} 1(\mathrm{~A} 2)$ is $A_{V} / N_{\mathrm{H}} \sim 2.6(3.2) \times 10^{-22} \mathrm{mag} \mathrm{cc^{2 }} \mathrm{H}^{-1}$ if the hydrogen column density in the sight line to $\mathrm{A} 1(\mathrm{~A} 2)$ is proportional to the surface mass density of the best-fit SIE with $z_{\mathrm{Y}}=z_{\mathrm{L}}$. The dust to gas mass ratio in the sight line to $\mathrm{A} 1(\mathrm{~A} 2)$ is also estimated as $\Sigma_{\text {dust }} / \Sigma_{\mathrm{H}} \sim 0.005(0.006)$ for the Galaxy dust model. These values are smaller than the Galactic values, but larger than those for LMC and SMC (Weingartner \& Draine 2001). Conversely, if we assume the dust mass from extinction and the dust-togas ratio for $\mathrm{SMC}$, the gas masses around $\mathrm{A} 1$ and $\mathrm{A} 2$ become much larger than those inferred from the best-fit SIE mass profile assuming that baryon traces dark matter. Therefore, we conclude that the dust to gas ratio and the dust properties of $\mathrm{Y}$ are much similar to those in Galaxy than to those in LMC or SMC if $\mathrm{Y}$ is associated with $\mathrm{H}_{\mathrm{I}}$ absorption systems at $z_{\mathrm{Y}} \sim z_{\mathrm{L}}$.

If $\mathrm{Y}$ is associated with another dwarf galaxy in the line of sight, the most probable redshift is $z_{\mathrm{Y}}=0.58$ at which the critical surface density is the smallest. Since the critical surface density is a broad function of redshift, the reasonable range of redshift is also broad. For instance, for a redshift of $0.28 \leq z_{\mathrm{Y}} \leq 1.0$, the difference in the critical surface density falls within 20 percent of the minimum value. This is consistent with our lensing constraint: $0.548 \leq z_{\mathrm{Y}} \leq 0.958$. Indeed, a recent theoretical analysis predicts that the expected convergence contribution from intervening structures is much larger than that from subhalos in MG 0414+0534 (Inoue 2016).

Although object Y seems to have a relatively large size $\gtrsim 5 \mathrm{kpc}$ for a galaxy with one-dimensional velocity dispersion of $\sim 30 \mathrm{~km} / \mathrm{s}$, the optical/NIR emission seems to be extremely small as $\lesssim 0.06 \mu \mathrm{Jy}$ at the $\mathrm{I}_{\text {band }}{ }^{6}$. If $\mathrm{Y}$ is at $z=0.5$, the corresponding stellar mass would be

6 The upper limit corresponds to $3 \sigma$ error in the I band flux (Falco et al. 1997).
$M_{\star} \lesssim 10^{6} M_{\odot}$ if the SED is similar to that of faint submillimeter galaxies like LESS 34 (Wiklind et al. 2014). However, it is unnatural unless huge amount of dust is hiding luminous stellar components. Perhaps, $\mathrm{Y}$ is an object like recently discovered ultra diffuse galaxies (UDGs) in Coma (van Dokkum et al. 2015; Koda et al. 2015). UDGs have stellar masses typical of dwarf galaxies but effective radii of Milky-Way sized objects. The stellar components may have been expanded due to outflow caused by starburst (Di Cintio et al. 2016) or AGN activity. Then the surface brightness in the optical/NIR band can be extremely small despite being gas-rich. The required high ellipticity $e_{\mathrm{Y}} \sim 0.7$ for $\mathrm{Y}$ is consistent with the shape of UDGs if prolate (Burkert 2016) and that of dark matter halos that host the Local Group dwarf spheroidal galaxies if oblate (Hayashi \& Chiba 2015). More detailed analysis of $\mathrm{Y}$ would give us a clue about the origin of past starburst activity. If $\mathrm{Y}$ is turned out to be residing in the intervening line of sight, then a substantial portion of faint submillimeter galaxies in the universe may be attributed to such gas-rich dusty dark dwarf galaxies.

KTI acknowledges support from the ALMA Japan Research Grant of NAOJ Chile Observatory, NAOJALMA-0110. SM is supported by the Ministry of Science and Technology (MoST) of Taiwan, MoST 103-2112-M-001-032-MY3. MC acknowledges support from MEXT Grant-in-Aid for Scientific Research on Innovative Areas (No. 15H05889, 16H01086). This paper makes use of the following ALMA data: ADS/JAO.ALMA\#2013.1.01110.S. ALMA is a partnership of ESO (representing its member states), NSF (USA) and NINS (Japan), together with NRC (Canada) and NSC and ASIAA (Taiwan), and KASI (Republic of Korea) in cooperation with the Republic of Chile. The Joint ALMA Observatory is operated by ESO, AUI/NRAO and NAOJ.

\section{REFERENCES}

Allison, J. R., Moss, V. A., Macquart, J.-P., et al. 2016, ArXiv e-prints, arXiv:1611.00863

Asplund, M., Grevesse, N., Sauval, A. J., \& Scott, P. 2009, ARA\&A, 47, 481

Barvainis, R., \& Ivison, R. 2002, ApJ, 571, 712

Burkert, A. 2016, ArXiv e-prints, arXiv:1609.00052

Chiba, M. 2002, ApJ, 565, 17

Chiba, M., Minezaki, T., Kashikawa, N., Kataza, H., \& Inoue, K. T. 2005, ApJ, 627, 53

Cornwell, T. J. 2008, IEEE Journal of Selected Topics in Signal Processing, 2, 793

Curran, S. J., Darling, J., Bolatto, A. D., et al. 2007, MNRAS, 382, L11

Dai, X., Kochanek, C. S., Chartas, G., \& Mathur, S. 2006, ApJ, 637,53 
Dalal, N., \& Kochanek, C. S. 2002, ApJ, 572, 25

Di Cintio, A., Brook, C. B., Dutton, A. A., et al. 2016, ArXiv e-prints, arXiv:1608.01327

Draine, B. T., \& Li, A. 2007, ApJ, 657, 810

Dunne, L., Eales, S., Edmunds, M., et al. 2000, MNRAS, 315, 115

Falco, E. E., Lehar, J., \& Shapiro, I. I. 1997, ApJ, 113, 540

Falco, E. E., Impey, C. D., Kochanek, C. S., et al. 1999, ApJ, 523,617

Gordon, K. D., Clayton, G. C., Misselt, K. A., Landolt, A. U., \& Wolff, M. J. 2003, ApJ, 594, 279

Hayashi, K., \& Chiba, M. 2015, ApJ, 810, 22

Hewitt, J. N., Turner, E. L., Lawrence, C. R., Schneider, D. P., \& Brody, J. P. 1992, ApJ, 104, 968

Hezaveh, Y. D., Dalal, N., Marrone, D. P., et al. 2016, ApJ, 823, 37

Inoue, K. T. 2015, MNRAS, 447, 1452

—. 2016, MNRAS, 461, 164

Inoue, K. T., \& Chiba, M. 2005a, ApJ, 634, 77

—. 2005b, ApJ, 633, 23

Inoue, K. T., Minezaki, T., Matsushita, S., \& Chiba, M. 2016, MNRAS, 457, 2936

Inoue, K. T., \& Takahashi, R. 2012, MNRAS, 426, 2978

Kochanek, C. S., \& Dalal, N. 2004, ApJ, 610, 69

Koda, J., Yagi, M., Yamanoi, H., \& Komiyama, Y. 2015, ApJL, 807, L2

Koopmans, L. V. E. 2005, MNRAS, 363, 1136

Kormann, R., Schneider, P., \& Bartelmann, M. 1994, Astronomy and Astrophysics, 284, 285

Lawrence, C. R., Elston, R., Januzzi, B. T., \& Turner, E. L. 1995, Astronomical Journal, 110, 2570

MacLeod, C. L., Jones, R., Agol, E., \& Kochanek, C. S. 2013, ApJ, 773, 35

Mao, S., \& Schneider, P. 1998, MNRAS, 295, 587
McMullin, J. P., Waters, B., Schiebel, D., Young, W., \& Golap, K. 2007, in Astronomical Society of the Pacific Conference Series, Vol. 376, Astronomical Data Analysis Software and Systems XVI, ed. R. A. Shaw, F. Hill, \& D. J. Bell, 127

Metcalf, R. B. 2005, ApJ, 629, 673

Metcalf, R. B., Moustakas, L. A., Bunker, A. J., \& Parry, I. R. 2004, ApJ, 607, 43

Metcalf, R. B., \& Zhao, H. 2002, ApJLetters, 567, L5

Minezaki, T., Chiba, M., Kashikawa, N., Inoue, K. T., \& Kataza, H. 2009, ApJ, 697, 610

More, A., McKean, J. P., More, S., et al. 2009, MNRAS, 394, 174

Oman, K. A., Navarro, J. F., Sales, L. V., et al. 2016, MNRAS, 460, 3610

Planck Collaboration, Ade, P. A. R., Aghanim, N., et al. 2016, A\&A, 594, A13

Ros, E., Guirado, J. C., Marcaide, J. M., et al. 2000, Astronomy and Astrophysics, 362, 845

Schechter, P. L., \& Moore, C. B. 1993, Astronomical Journal, 105,1

Sugai, H., Kawai, A., Shimono, A., et al. 2007, ApJ, 660, 1016

Takahashi, R., \& Inoue, K. T. 2014, MNRAS, 440, 870

Tonry, J. L., \& Kochanek, C. S. 1999, Astronomical Journal, 117, 2034

Trotter, C. S., Winn, J. N., \& Hewitt, J. N. 2000, ApJ, 535, 671

van Dokkum, P. G., Abraham, R., Merritt, A., et al. 2015, ApJL, 798, L45

Vegetti, S., Koopmans, L. V. E., Auger, M. W., Treu, T., \& Bolton, A. S. 2014, MNRAS, 442, 2017

Vegetti, S., Lagattuta, D. J., McKean, J. P., et al. 2012, Nature, 481,341

Weingartner, J. C., \& Draine, B. T. 2001, ApJ, 548, 296

Wiklind, T., Conselice, C. J., Dahlen, T., et al. 2014, ApJ, 785, 111

Xu, D. D., Mao, S., Cooper, A. P., et al. 2012, MNRAS, 421, 2553

—. 2010, MNRAS, 408, 1721

Xu, D. D., Mao, S., Wang, J., et al. 2009, MNRAS, 398, 1235 